\title{
TITLE:
}

\section{Can distributed delays perfectly stabilize dynamical networks?}

$\operatorname{AUTHOR}(S)$ :

Omi, Takahiro; Shinomoto, Shigeru

CITATION:

Omi, Takahiro ...[et al]. Can distributed delays perfectly stabilize

dynamical networks?. Physical Review E 2008, 77(4)

ISSUE DATE:

2008-04

URL:

http://hdl.handle.net/2433/140399

RIGHT:

(C)2008 The American Physical Society 
PHYSICAL REVIEW E 77, 046214 (2008)

\title{
Can distributed delays perfectly stabilize dynamical networks?
}

\author{
Takahiro Omi* and Shigeru Shinomoto ${ }^{\dagger}$ \\ Department of Physics, Kyoto University, Sakyo-ku, Kyoto 606-8502, Japan \\ (Received 18 October 2007; revised manuscript received 12 February 2008; published 17 April 2008)
}

\begin{abstract}
Signal transmission delays tend to destabilize dynamical networks leading to oscillation, but their dispersion contributes oppositely toward stabilization. We analyze an integrodifferential equation that describes the collective dynamics of a neural network with distributed signal delays. With the $\Gamma$ distributed delays less dispersed than exponential distribution, the system exhibits reentrant phenomena, in which the stability is once lost but then recovered as the mean delay is increased. With delays dispersed more highly than exponential, the system never destabilizes.
\end{abstract}

DOI: 10.1103/PhysRevE.77.046214

PACS number(s): 05.45.Xt, 02.30.Ks, 87.18.Sn

\section{INTRODUCTION}

Computational capability of neural networks had been discussed utilizing the robust stability achieved with the cooperative interaction among neurons. In model networks, the stability can easily be obtained by introducing the delayless symmetrical connections between neurons [1,2]. However, the transmission delays unavoidably present in hard-wired networks may destabilize the system causing the oscillation or chaos [3-6]. On the other hand, the distribution of delays contributes toward stabilization, in neural networks $[7,8]$, ecological systems $[9,10]$, control engineering $[11,12]$, biology [13], or coupled dynamical systems [14-18]. In the brain, the signal transmission delays are widely distributed in time [19-23].

In the present paper, we wish to fully comprehend how the macroscopic stability of dynamical systems is controlled by these competing factors; the mean and the dispersion of delays. For this purpose, we examine the stability of an integrodifferential equation $[13,24,25]$ derived from microscopic dynamics of a neural network whose signal transmission delays are distributed in time. It is revealed from the analysis that the network with $\Gamma$ distributed delays less dispersed than exponential distribution exhibits reentrant stability; the system once destabilizes but then recovers the stability as the average delay is increased. With delays dispersed more highly than exponential distribution, the system attains a perfect macroscopic stationarity.

This paper is organized as follows. In Sec. II a dynamical equation for the macroscopic order parameter is derived. In Sec. III the linear stability of the steady state for the macroscopic dynamical equation is analyzed. In Sec. IV the raw macroscopic equation is analyzed numerically for several delay distributions. In Sec. V the condition for the perfect stability is sought. In Sec. VI the microscopic dynamics are numerically simulated to compare with the result of the macroscopic equation. In Sec. VII the effect of the distributed delays for the occurrence of macroscopic oscillation is discussed.

\footnotetext{
*omi@ton.scphys.kyoto-u.ac.jp

† shinomoto@scphys.kyoto-u.ac.jp
}

\section{DERIVATION OF A MACROSCOPIC STATE EQUATION}

We consider a network of model neurons that obey the evolution equation

$$
\tau \frac{d x_{i}(t)}{d t}=-x_{i}(t)+\operatorname{sgn}\left(\sum_{j=1}^{n} w_{i, j} x_{j}\left(t-d_{i, j}\right)+s_{i}\right),
$$

where $\operatorname{sgn}(v)$ is the sign function that takes values +1 and -1 , respectively, for $v>0$ and $v \leq 0 . \tau$ is the "membrane time constant" of an individual neuron. The "synaptic weight" $w_{i, j}$ and the signal transmission delay $d_{i, j}$ are fixed to each transmission line from $j$ th neuron to $i$ th neuron (Fig. 1). $s_{i}$ is the "external stimulus" to each neuron.

A dynamical equation of the macroscopic order parameter $X(t) \equiv \frac{1}{n} \sum_{i=1}^{n} x_{i}(t)$ can be derived in a manner similar to what we have done for the discrete time model [8] in parallel with Amari's derivation for the synchronous update rule [26-29]: A mean field exerted on $X(t)$ is given by the difference of ratios of positive and negative inputs. Using the distribution $p_{t}(v)$ of inputs $\left\{v_{i}=\sum_{j=1}^{n} w_{i, j} x_{j}\left(t-d_{i, j}\right)+s_{i}\right\}$ at time $t$, the macroscopic equation can be represented as

$$
\tau \frac{d X(t)}{d t}=-X(t)+\int_{0}^{\infty} p_{t}(v) d v-\int_{-\infty}^{0} p_{t}(v) d v .
$$

Under the assumption of the statistical independence of $\left\{x_{j}\left(t-d_{i, j}\right)\right\}$ from $\left\{w_{i, j}\right\}$, the central limit theorem holds for the summed inputs $\left\{\sum_{j=1}^{n} w_{i, j} x_{j}\left(t-d_{i, j}\right)\right\}$. If in addition $\left\{s_{i}\right\}$ are normally distributed, the distribution $p_{t}(v)$ can be approximated as Gaussian characterized solely by the mean and the variance at time $t$. Then, the macroscopic state equation is obtained as

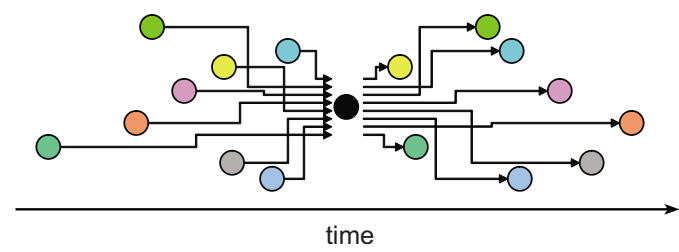

FIG. 1. (Color online) Schematic diagram of distributed signal transmission delays. 


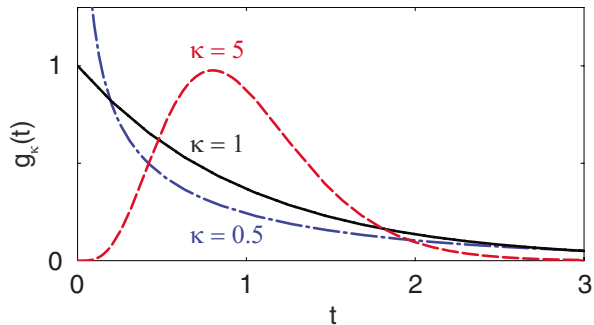

FIG. 2. (Color online) The $\Gamma$ distributions of identical means $(T=1)$ with different shape factors: Exponentially distributed $(\kappa=1)$, with the standard deviation identical to the mean; more dispersed $(\kappa<1)$, with the standard deviation greater than the mean; less dispersed $(\kappa>1)$, with the standard deviation smaller than the mean.

$$
\tau \frac{d X(t)}{d t}=-X(t)+F(I)
$$

where $F(x)$ is the error function

$$
F(I)=\sqrt{\frac{2}{\pi}} \int_{0}^{I} e^{-x^{2} / 2} d x,
$$

and $I$ is the ratio of the mean and the standard deviation of inputs $\left\{v_{i}\right\}$ to neurons

$$
I=\frac{n \mu_{w}\langle x\rangle+\mu_{s}}{\sqrt{n\left(\sigma_{w}^{2}+\mu_{w}^{2}\right)\left\langle x^{2}\right\rangle-n \mu_{w}^{2}\langle x\rangle^{2}+\sigma_{s}^{2}}},
$$

where $\mu_{w}, \mu_{s}, \sigma_{w}^{2}$, and $\sigma_{s}^{2}$ are, respectively, the means and variances of $\left\{w_{i, j}\right\}$ and $\left\{s_{j}\right\}$.

Under the assumption that microscopic states $\left\{x_{i}\right\}$ are statistically independent from delays $\left\{d_{i, j}\right\}$, the mean past activity $\langle x\rangle \equiv \frac{1}{n^{2}} \sum_{i=1}^{n} \sum_{j=1}^{n} x_{j}\left(t-d_{i, j}\right)$ can be represented by the macroscopic order parameter [8] as

$$
\langle x\rangle \equiv \frac{1}{n^{2}} \sum_{i=1}^{n} \sum_{j=1}^{n} x_{j}\left(t-d_{i, j}\right)=\int_{0}^{\infty} g(s) X(t-s) d s,
$$

where $g(s)$ represents the distribution of delays. $\left\langle x^{2}\right\rangle$ $\equiv \frac{1}{n^{2}} \sum_{i=1}^{n} \sum_{j=1}^{n} x_{j}^{2}\left(t-d_{i, j}\right)$ remains close to unity, if individual microscopic states are always approaching swiftly either of \pm 1 .

If $n\left(\sigma_{w}^{2}+\mu_{w}^{2}\right) \ll \sigma_{s}^{2}$, or if $\left\langle x^{2}\right\rangle$ can be approximated as unity, then the dynamical equation (3) is closed with the macroscopic order parameter $X(t)$. Furthermore, if the model parameters satisfy $n \mu_{w}^{2} \ll n \sigma_{w}^{2}+\sigma_{s}^{2}$, the evolution equation simplifies to

$$
\tau \frac{d X(t)}{d t}=-X(t)+F\left(W \int_{0}^{\infty} g(s) X(t-s) d s+S\right),
$$

where $W=n \mu_{w} / \sqrt{n \sigma_{w}^{2}+\sigma_{s}^{2}}$ and $S=\mu_{s} / \sqrt{n \sigma_{w}^{2}+\sigma_{s}^{2}}$. Note that $n \mu_{w}^{2} \ll n \sigma_{w}^{2}+\sigma_{s}^{2}$ is not an essential condition for a macroscopic equation (7) to hold but is merely introduced to make the analysis simpler.

The Eq. (7) is similar to the macroscopic equation derived by Wilson and Cowan [30], and also to the firing rate neuron model with synaptic filtering [31]. These conventional stud- ies have reduced the integrodifferential equation into a delayless differential equation assuming that signal transmission delays are sufficiently short in comparison to the time scale of single neuron dynamics. The oscillation may emerge even in the delayless system due to the interaction between the groups of excitatory and inhibitory neurons.

In the present study, we accept the fact that signal transmission delays in biological networks are widely distributed in time, and would like to examine the cases in which the distributed delays play a central role in the dynamics $[7,8,32-34]$. We directly treat this integrodifferential equation (7) and investigate the influence of the dispersed delays on the macroscopic stability of the system. We adopt here the $\Gamma$ distributed delays

$$
g(s)=\frac{\kappa}{\Gamma(\kappa) T}\left(\frac{\kappa s}{T}\right)^{\kappa-1} \exp \left(-\frac{\kappa s}{T}\right),
$$

which is characterized by the scale factor $T$ representing the average delay, and the shape factor $\kappa$ representing (inversely related to) the dispersion of delays (Fig. 2). $\Gamma(\kappa)$ is the $\Gamma$ function defined by $\int_{0}^{\infty} x^{\kappa-1} e^{-x} d x$.

\section{LINEAR STABILITY ANALYSIS OF MACROSCOPIC STATIONARY STATES}

In this section, we perform the linear stability analysis of the macroscopic Eq. (7). In the absence of the transmission delay, the system is perfectly stable for an arbitrary set of model parameters. The system may be destabilized by transmission delays, causing oscillation. We examine how the parametric range for oscillation varies with the scale factor representing the mean transmission delay and the shape parameter representing the dispersion of delays.

The macroscopic Eq. (7) is linearized with respect to the deviation from a macroscopic stationary state $X(t)=X_{0}$ that satisfies $X_{0}=F\left(W X_{0}+S\right)$. The characteristic equation for the linearized integrodifferential equation is obtained by putting $X(t)-X_{0}=\exp (\lambda t / \tau)$ as

$$
(1+\lambda)(1+\lambda T / \tau \kappa)^{\kappa}=\beta,
$$

where $\beta$ represents the slope of the response function,

$$
\left.\beta \equiv \frac{d F(W X+S)}{d X}\right|_{X=X_{0}} .
$$

For positive $\beta$, the system exhibits instability $\lambda \geq 0$ if the response function $F(W X+S)$ has a slope greater than unity, $\beta>1$. In this case, the system eventually attains one of stable stationary states due to the nonlinear saturation.

A dynamical instability leading to oscillation may take place for negative $\beta$. In this case, the linear-stability boundary is obtained by solving a pair of simultaneous equations that represent the condition for the characteristic equation (9) to have a pure imaginary solution $\lambda=i \omega$ :

$$
\begin{gathered}
\arctan (\omega)+\kappa \arctan (T \omega / \tau \kappa)=\pi, \\
\beta^{2}=\left(1+\omega^{2}\right)\left[1+(T \omega / \tau \kappa)^{2}\right]^{\kappa} .
\end{gathered}
$$

Equation (11) has a solution only if $\kappa>1$. In the limit of $T / \tau \rightarrow 0, \omega$ is obtained as 


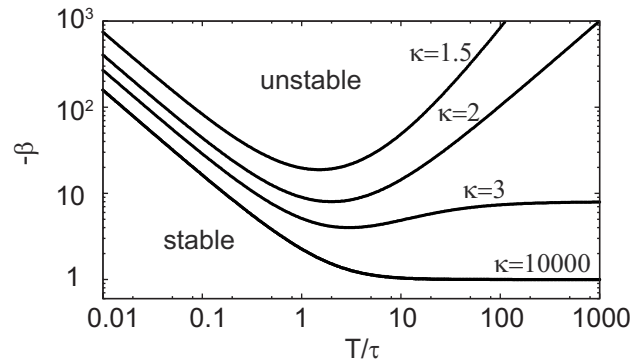

FIG. 3. The stability boundaries in the space of $\{T / \tau,-\beta\}$ obtained for several shape parameters $\kappa=1.5,2,3,10000$.

$$
\omega \approx(\kappa \tau / T) \tan (\pi / 2 \kappa),
$$

and critical $\beta$ is obtained from Eq. (12) as

$$
\beta \approx-(\kappa \tau / T) \tan (\pi / 2 \kappa)\left[1+\tan ^{2}(\pi / 2 \kappa)\right]^{\kappa / 2} .
$$

In the opposite extreme of $T / \tau \rightarrow \infty, \omega$ is obtained as

$$
\begin{gathered}
\omega \approx(\kappa \tau / T) \tan (\pi / \kappa), \quad(\kappa>2), \\
\omega \approx \tan [\pi(1-\kappa / 2)],(2>\kappa>1),
\end{gathered}
$$

with which the critical $\beta$ is obtained, respectively, as

$$
\begin{gathered}
\beta \approx-\left[1+\tan ^{2}(\pi / \kappa)\right]^{\kappa / 2},(\kappa>2), \\
\beta \approx-\{(T / \tau \kappa) \tan [\pi(1-\kappa / 2)]\}^{\kappa} \\
\times\left\{1+\tan ^{2}[\pi(1-\kappa / 2)]\right\}^{1 / 2},(2>\kappa>1) .
\end{gathered}
$$

Figure 3 depicts the stability boundaries in the phase space of $\{T / \tau,-\beta\}$ obtained by numerically solving Eqs. (11) and (12) for several shape parameters $\kappa$ : With identical delays $(\kappa \rightarrow \infty)$, the critical coupling strength $|\beta|$ decreases monotonically with the average delay $T$. In other words, the system becomes more fragile with delays. This fact is consistent with the knowledge that the transmission delay is a destabilizing factor. It is notable that a system with dispersed delays exhibits reentrant phenomena; the stability is once lost but then recovered as the average delay $T$ is increased. With the delays of small dispersion $(2<\kappa<\infty)$, the critical $|\beta|$ rebounds and then saturates to a finite value. In a middle range of dispersion $(1<\kappa \leq 2)$, the critical $|\beta|$ takes a minimum and diverges with $T$. With the delays highly dispersed $(\kappa$ $\leq 1)$, the network never destabilizes.

In most cases, the critical $|\beta|$ increases with the dispersion of delays $1 / \kappa$. However, there are cases in which the critical $|\beta|$ decreases with the dispersion. Figure 4 depicts how the critical $|\beta|$ depends on the dispersion of delays $1 / \kappa$, for various mean delays $T$.

\section{NUMERICAL ANALYSIS OF THE MACROSCOPIC STATE EQUATION}

Next, we numerically solve the macroscopic evolution equation (7) to see if there is nontrivially coexisting oscillation or chaos in the parameter range in which the linear stability is confirmed. For this purpose, we have tested a number of random initial conditions of various power spectra;

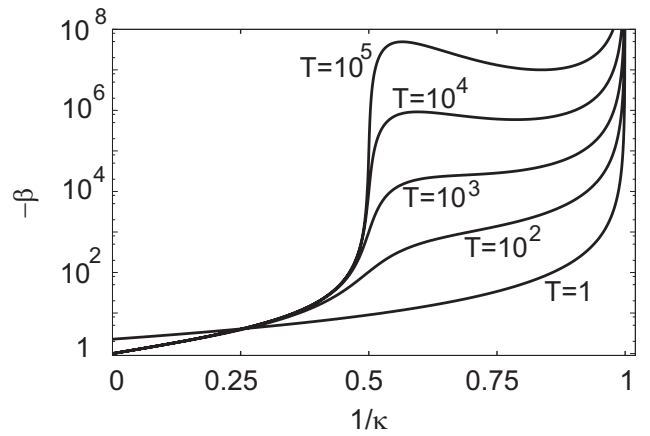

FIG. 4. The dependences of critical $-\beta$ on the dispersion of delays $1 / \kappa$.

from white (jagged) to colored (smooth) random temporal patterns.

Solving an integrodifferential equation is generally a hard computational task. In some particular conditions, however, the computational complexity can be reduced drastically by devising efficient algorithms: For the exponential distribution of delays $(\kappa=1)$, the mean past activity $\int_{0}^{\infty} e^{-s} X(t-s) d s$ is represented by

$$
A_{t}=\sum_{j=0}^{\infty} e^{-j \Delta} X(t-j \Delta),
$$

where $\Delta$ is a unit step. Due to the exponential kernel, $A_{t}$ can be obtained by simply iterating the recurrence equation

$$
A_{t}=e^{-\Delta} A_{t-\Delta}+X(t) \times \Delta .
$$

In addition, for the case of $\kappa=2$, the computational complexity could be reduced by utilizing the relation of

$$
t e^{-t} \approx \frac{e^{-t}-e^{-(1+\varepsilon) t}}{\epsilon},
$$

with sufficiently small $|\epsilon|$.

To the extent we have examined, we have not found any nontrivial coexisting dynamical orbits in the parameter range that the linear stability is guaranteed. Figure 5 shows an amplitude of $X(t)$ obtained for the systems with shape parameters of $\kappa=2$ and 1 . In the case of $\kappa=2$, a significant ampli-

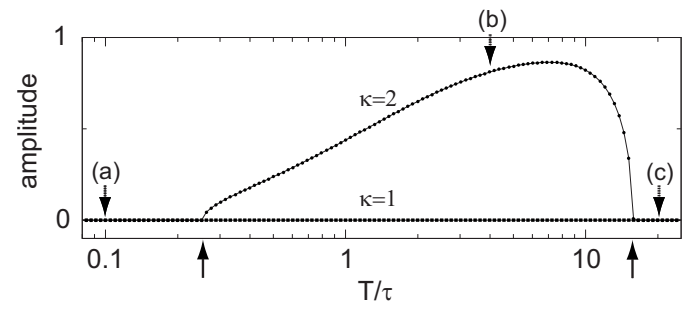

FIG. 5. The amplitude of $X(t)$ numerically obtained for $W=$ -25 and $S=0,(\beta=-20)$; The linear instability boundaries for $\kappa=2$ are $T / \tau=0.254$ and 15.7 and depicted by the arrows. They coincide with the critical points at which the system numerically shows reentrant stability; The system with delays exponentially distributed $(\kappa=1)$ always remains stable. The dynamics of the order parameter $X(t)$ for $\kappa=2$. (a) $T=0.1$, (b) $T=4$, (c) $T=20$ will be illustrated in Fig. 8. 


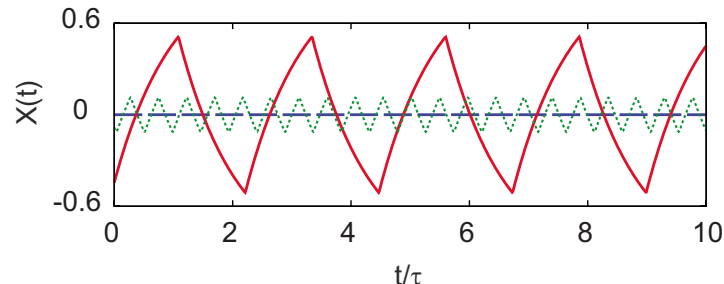

FIG. 6. (Color online) The macroscopic order parameters $X(t)$ numerically obtained for the cases of $W=-1250$ and $S=0,(\beta$ $=-1000)$, and $T / \tau=1$. Solid line: Oscillation observed for the shape parameter $\kappa=2$. Dashed line: Stability observed for $\kappa=1$. Dotted line: Rapid oscillation of small amplitude observed for the $\Gamma$ distributed delays of $\kappa=1$ accompanied by a time lag of $\epsilon / \tau=0.01$.

tude of $X(t)$ can be observed in the interval of mean delay $T$ in which the system is linearly unstable. For the shape parameter $\kappa=1$ with which the system is linearly stable, the amplitude is negligibly small in the whole range of $T$.

\section{VARIOUS DELAY DISTRIBUTIONS}

What is the key factor in the perfect stability? As the system shows a perfect stability in the absence of delay, we suspect if a fraction of instantaneous signal transmissions lead to the stability for $\kappa \leq 1$. We examine the stability of a system with the delay distribution composed of two delta functions peaked at zero and finite delays $a \delta(t)+(1-a) \delta(t$ $-T)$. It is found from the characteristic equation that the system can be destabilized even if delayless lines are present in a finite fraction $0 \leq a<1 / 2$. This fact demonstrates that the presence of instantaneous signal transmissions alone does not necessarily induce a perfect stability.

Next, we suspect if the long tail of the delay distribution has led to the stability. We examine whether or not a system remains stable even if a lag is added to $\Gamma$ distributed delays

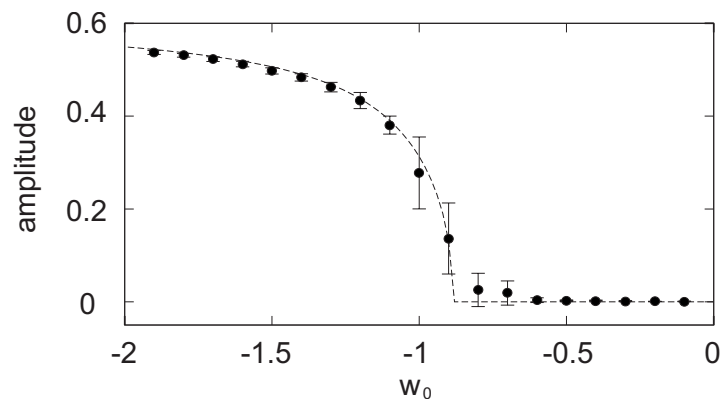

FIG. 7. Emergence of macroscopic oscillation. The dashed line represents the amplitude of the order parameter evaluated by numerically solving the mean-field equation (7) with parameters $\kappa$ $=5, T=1, W=w_{0} 5$, and $S=0$. Error bars represent the distributions of the order parameter amplitudes obtained from simulating twenty networks of $n=500$ neurons whose microscopic parameters were independently sampled: External stimuli to individual neurons fixed in time were drawn from the Gaussian distribution of the mean $\mu_{s}=0$ and variance $\sigma_{s}^{2}=10000$. The synaptic couplings and the time scale of the individual dynamics were set uniform: $w_{i j}=w_{0}$ and $\tau$ $=1$. The delay intervals $\left\{d_{i j}\right\}$ were derived from the $\Gamma$ distribution of $\kappa=5$ with the mean $T=1$. (a) $\mathrm{T}=0.1$
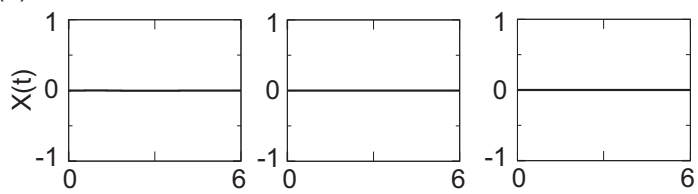

(b) $\mathrm{T}=4$
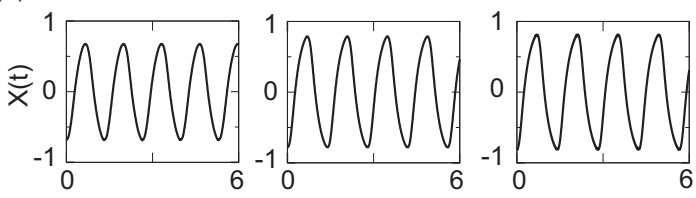

(c) $\mathrm{T}=20$
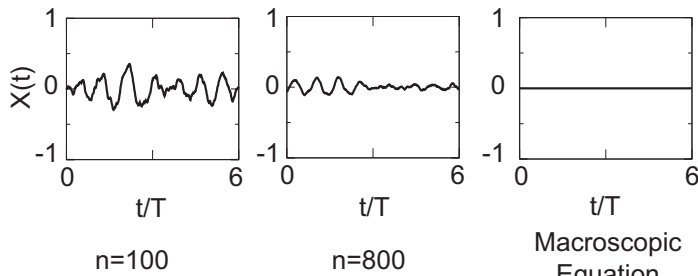

FIG. 8. Order parameter dynamics obtained with the numerical simulations of the networks of $n=100$ (left) and $n=800$ (middle), in reference to that of the macroscopic equation (right). The microscopic parameters $n=100, \mu_{s}=0, \sigma_{s}=4, w_{0}=-1, \kappa=2$ (left) and $n=800, \mu_{s}=0, \sigma_{s}=32, w_{0}=-1, \kappa=2$ (middle) correspond to the parameters of macroscopic equation $W=-25, S=0, g(x): \kappa=2$ (right). Simulations for (a) were carried out with distributed delays of the mean $T=0.1$. The individual delays $\left\{d_{i j}\right\}$ were increased from (a) by 40 for (b) and by 200 for (c).

(8) as $\theta(t-\epsilon) g(t-\epsilon)$, where $\theta(x)$ is the Heaviside step function $\theta(x)=1$ if $x \geq 0$ and $=0$ otherwise. In the presence of a lag $\epsilon>0$, the instability condition equation (11) is modified as

$$
\arctan (\omega)+\kappa \arctan (T \omega / \tau \kappa)+\epsilon \omega / \tau=\pi .
$$

This characteristic equation possesses a solution even for the case of a high dispersion $\kappa \leq 1$. This implies that the perfect stability can be destroyed by a time lag. As long as the time lag $\epsilon$ is small, however, the system exhibits an instability at a very high frequency of the order of $\tau \pi / \epsilon$, and the amplitude of the order parameter $X(t)$ cannot grow large due to the nonlinearity of the system. This point is verified by directly solving the original nonlinear macroscopic evolution equation. Figure 6 compares the order parameters $X(t)$ computed for the several kinds of delay distributions: the system of $\kappa$ $=2$ may exhibit a large amplitude $X(t)$; the system of $\kappa=1$ has never yielded a significant amplitude $X(t)$; the system with $\Gamma$ distributed delays of $\kappa=1$ accompanied by a small time lag $\epsilon / \tau=0.01$ has yielded an order parameter $X(t)$ rapidly oscillating with a small amplitude.

\section{NUMERICAL SIMULATION OF MICROSCOPIC DYNAMICS}

In this section, the microscopic evolution equation (1) is numerically simulated to examine the validity of the meanfield 
approximation we employed in deriving the macroscopic equation by comparing the network dynamics with the solution of the mean-field equation (7). In Fig. 7, the distributions of the order parameter amplitudes obtained by simulating twenty networks of $n=500$ neurons are compared to the solution of the macroscopic Eq. (7). The agreement between these results demonstrated in Fig. 7 confirms the numerical validity for the mean field approximation employed in deriving the macroscopic equation.

Next, the reentrant phenomenon of the macroscopic oscillation is examined with the numerical simulation of the microscopic equation. Figure 8 compares the temporal dynamics of the order parameter for the networks of $n=100$ (left) and $n=800$ (middle), in reference to that of the macroscopic equation (right). The mean delay $T$ is increased from the top (a) to the bottom (c) in Fig. 8 so that the network parameters become identical to three points (a), (b), and (c) in Fig. 5. By increasing $T$ from (a) to (b), the macroscopic oscillation emerges. By increasing the mean delay further to (c), the stable macroscopic oscillation disappears: Although some noisy fluctuation remains in the smaller network of $n=100$, it is less prominent in the larger network $n=800$, implying the reentrant stability predicted by the macroscopic equation.

\section{DISCUSSION}

In the present study, we examined the stability of a neural network whose signal transmission delays are distributed in time. The network is found to exhibit a reentrant stability for the delays less dispersed than the exponential distribution. The network attains a perfect stability for the highly dispersed delays.
We should like to compare the present reentrant phenomenon with others [35-37]. For instance, the network examined by Gerstner consists of spiking neurons that oscillate autonomously, and exhibits rich reentrance of the macroscopic coherence. In the systems of this kind, abundant phases are yielded by the interaction between the native oscillation and the delay. In contrast, the present study adopted passive elements that do not exhibit autonomous oscillation, and the whole network neither exhibits oscillation in the absence of transmission delays. In the present network, the oscillatory instability is induced genuinely by the delays and the stability is recovered due to their dispersion. Thus the mechanisms of the reentrant phenomena are totally different.

Next, we should like to note that the perfect stability due to the dispersion was indicated by Eurich et al. [10]. They proved the perfect stability in the limit of highest dispersion $\kappa \rightarrow 0$ in an ecological feedback system. In the present paper, we have revealed that the perfect stability can be manifested not only in the limit, but also in a finite range of the dispersion or the shape parameter $0<\kappa \leq 1$ for a neural network. It is desirable to examine the generality of the present findings; whether or not the perfect stability is achieved solely due to the delay distribution, irrespective of detailed dynamics of individual elements.

\section{ACKNOWLEDGMENTS}

This study was supported in part by Grants-in-Aid for Scientific Research to S.S. from MEXT Japan (Grants No. 16300068 , No. 18020015), and by the 21st century COE "Center for Diversity and Universality in Physics."
[1] M. A. Cohen and S. Grossberg, IEEE Trans. Syst. Man Cybern. 13, 815 (1983).

[2] J. J. Hopfield, Proc. Natl. Acad. Sci. U.S.A. 81, 3088 (1984).

[3] M. C. Mackey and L. Glass, Science 197, 287 (1977).

[4] K. Ikeda and K. Matsumoto, Physica D. 29, 223 (1987).

[5] C. M. Marcus and R. M. Westervelt, Phys. Rev. A 39, 347 (1989).

[6] K. Gopalsamy and X. He, Physica D. 76, 344 (1994).

[7] P. Gong and C. van Leeuwen, Phys. Rev. Lett. 98, 048104 (2007).

[8] T. Omi and S. Shinomoto, Phys. Rev. E 76, 051908 (2007).

[9] A. Thiel, H. Schwegler, and C. W. Eurich, Complexity 8, 102 (2003).

[10] C. W. Eurich, A. Thiel, and L. Fahse, Phys. Rev. Lett. 94, 158104 (2005).

[11] J. E. S. Socolar and D. J. Gauthier, Phys. Rev. E 57, 6589 (1998).

[12] A. Ahlborn and U. Parlitz, Phys. Rev. Lett. 93, 264101 (2004).

[13] N. MacDonald, Biological Delay Systems: Linear Stability Theory (Cambridge University Press, New York, 1989).

[14] F. M. Atay, Phys. Rev. Lett. 91, 094101 (2003).

[15] V. K. Jirsa and M. Ding, Phys. Rev. Lett. 93, 070602 (2004).

[16] C. Masoller and A. C. Marti, Phys. Rev. Lett. 94, 134102 (2005).
[17] A. C. Marti, M. Ponce, and C. Masoller, Phys. Rev. E 72, 066217 (2005)

[18] D. Huber and L. S. Tsimring, Phys. Rev. E 71, 036150 (2005).

[19] H. A. Swadlow, J. Neurophysiol. 54, 1346 (1985).

[20] V. Bringuier, F. Chavane, L. Glaeser, and Y. Frégnac, Science 283, 695 (1999).

[21] P. Girard, J. M. Hupé, and J. Bullier, J. Neurophysiol. 85, 1328 (2001).

[22] J. G. Pelletier and D. Paré, J. Neurophysiol. 87, 1213 (2002).

[23] A. F. Soleng, M. Raastad, and P. Andersen, Hippocampus 13, 953 (2003).

[24] M. N. Oğuztöreli, Time-Lag Control Systems (Academic Press, New York, 1966).

[25] J. K. Hale and S. M. V. Lunel, Introduction to Functional Differential Equations (Springer-Verlag, New York, 1993).

[26] S. Amari, Proc. IEEE 59, 35 (1971).

[27] W. Kinzel, Z. Phys. B: Condens. Matter 60, 205 (1985).

[28] S. Shinomoto, Prog. Theor. Phys. 75, 1313 (1986).

[29] S. Shinomoto, Biol. Cybern. 57, 197 (1987).

[30] H. R. Wilson and J. D. Cowan, Biophys. J. 12, 1 (1972).

[31] P. Dayan and L. F. Abbott, Theoretical Neuroscience (MIT Press, Cambridge, 2001).

[32] E. M. Izhikevich, Neural Comput. 18, 245 (2006). 
[33] A. Hutt and F. M. Atay, Phys. Rev. E 73, 021906 (2006).

[34] T. W. Ko and G. B. Ermentrout, Phys. Rev. E 76, 056206 (2007)

[35] W. Gerstner and J. L. van Hemmen, Phys. Rev. Lett. 71, 312
(1993).

[36] W. Gerstner, Neural Comput. 12, 43 (2000).

[37] M. K. Stephen Yeung and S. H. Strogatz, Phys. Rev. Lett. 82, 648 (1999). 